\title{
To What Degree Do Pain-coping Strategies Affect Joint Stiffness and Functional Outcomes in Patients with Hand Fractures?
}

\author{
Young Hak Roh MD, Jung Ho Noh MD, \\ Joo Han Oh MD, Hyun Sik Gong MD, \\ Goo Hyun Baek MD
}

Published online: 31 March 2015

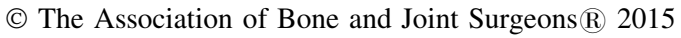

\begin{abstract}
Background Patients with hand fractures often have pain, swelling, and stiffness in the joints of the hand, which may lead them to protect their hands, resulting in more stiffness and in delayed recovery. However, the effects of paincoping strategies and catastrophization (the tendency to expect the worst to occur when pain is present, an approach that can be thought of as the opposite of "coping") on
\end{abstract}

Each author certifies that he or she, or a member of their immediate family, has no commercial associations (eg, consultancies, stock ownership, equity interest, patent/licensing arrangements, etc) that might pose a conflict of interest in connection with the submitted article.

All ICMJE Conflict of Interest Forms for authors and Clinical Orthopaedics and Related Research ${ }^{\mathbb{R}}$ editors and board members are on file with the publication and can be viewed on request. Clinical Orthopaedics and Related Research ${ }^{\mathbb{R}}$ neither advocates nor endorses the use of any treatment, drug, or device. Readers are encouraged to always seek additional information, including FDAapproval status, of any drug or device prior to clinical use.

Each author certifies that his or her institution approved the human protocol for this investigation, that all investigations were conducted in conformity with ethical principles of research, and that informed consent for participation in the study was obtained.

\section{Y. H. Roh}

Department of Orthopaedic Surgery, Gil Medical Center,

Gachon University School of Medicine, Incheon, Korea

\section{J. H. Noh (ه)}

Department of Orthopaedic Surgery, Kangwon National University Hospital, 156 Baengnyeong-ro, Chuncheon-si, Gangwon-do 200-722, Korea

e-mail: bestknee@hotmail.com

J. H. Oh, H. S. Gong, G. H. Baek

Department of Orthopaedic Surgery, Seoul National University

College of Medicine, Seoul, Korea functional recovery after hand fractures have not been investigated in depth.

Questions/purposes Are preoperative catastrophization and anxiety in patients with hand fractures associated with (1) decreased grip strength; (2) decreased range of motion; and (3) increased disability at 3 and 6 months after surgical treatment for a hand fracture? Secondarily, we asked if there are other patient and injury factors that are associated with these outcomes at 3 and 6 months.

Methods A total of 93 patients with surgically treated hand fractures were enrolled in this prospective study. Preoperative assessments measured coping strategies evaluated by measuring catastrophic thinking with the Pain Catastrophizing Scale and pain anxiety with the Pain Anxiety Symptom Scale. At 3 and 6 months postoperatively, grip strength, total active range of motion, and disability (Quick Disabilities of the Arm, Shoulder, and Hand score) were assessed. Bivariate and multivariate analyses were performed to identify patient demographic, injury, and coping skills factors that accounted for outcomes of strength, motion, and disability.

Results Decreased grip strength was associated with catastrophic thinking (beta $=-1.29$ [95\% confidence interval, -1.67 to -0.89$]$, partial $\left.\mathrm{R}^{2}=11 \%, \mathrm{p}<0.001\right)$ and anxiety (beta $=-0.83[-1.16$ to -0.50$]$, partial $\mathrm{R}^{2}=7 \%$, $\mathrm{p}=0.007)$ at 3 months, but by 6 months, only anxiety (beta $=-0.74 \quad[-1.04$ to -0.44$]$, partial $\mathrm{R}^{2}=7 \%$, $\mathrm{p}=0.010$ ) remained an important factor. Decreased total active range of motion was associated with pain catastrophizing (beta $=-0.63[-0.90$ to -0.36$]$, partial $\mathrm{R}^{2}=6$ $\%, \mathrm{p}=0.024$ ) and anxiety (beta $=-0.28[-0.42$ to $-0.14]$, partial $\mathrm{R}^{2}=3 \%, \mathrm{p}=0.035$ ) at 3 months but not at 6 months. Similarly, increased disability was associated with pain catastrophizing (beta $=1.09$ [1.39-0.79], partial $\mathrm{R}^{2}=12 \%, \mathrm{p}<0.001$ ) and anxiety (beta $=0.93[1.21-$ 
0.65], partial $\mathrm{R}^{2}=11 \%, \mathrm{p}=0.001$ ) at 3 months; these factors failed to be associated for 6-month outcomes.

Conclusions Preoperative poor coping skills as measured by high catastrophization and anxiety were associated with a weaker grip strength, decreased range of motion, and increased disability after surgical treatment for a hand fracture at 3 months. However, poor coping skills did not show persistent effects beyond 6 months. More research may be needed to show interventions to improve coping skills will enhance treatment outcome in patients after acute hand fractures.

Level of Evidence Level III, prognostic study.

\section{Introduction}

Psychological distress related to pain depends not only on the individual's awareness of the level of threat represented by the injury, but also the choice of coping strategies [24]. The patient's coping strategies such as catastrophization (the tendency to expect the worst to occur when pain is present, an approach that can be thought of as the opposite of "coping") and pain anxiety are normal protective responses to initial nociception [10], but patients who depend on maladaptive and ineffective strategies to cope with pain often become impaired by their pain and maintain an inactive lifestyle [25]. For instance, patients with hand fractures often have pain, swelling, and stiffness in the joints of the hand, which may lead them to protect their hands, resulting in more stiffness and in delayed recovery [3]. Pain-coping strategies have been considered to be key factors that explain the magnitude of a disability or the intensity of pain for a painful illnesses [16, 25], and maladaptive nociception (defined as disabling misconception regarding pain) with overcautious activity restriction has been associated with posttraumatic stiffness and delayed recovery in hand fractures [10].

However, the effects of pain-coping strategies on functional recovery after hand fractures have not been investigated in depth. Prior studies demonstrated the effect of pain-coping strategies on patient-perceived disability in a cross-sectional study design without considering the context of functional recovery after acute hand injuries [3, 10]. In addition, the relationships between ineffective coping strategies and treatment outcome have not been comprehensively evaluated by both a self-report and objective measures of hand function.

We hypothesized that preoperative catastrophization and anxiety in patients with hand fractures would be associated with a (1) decrease in grip strength; (2) decreased ROM; and (3) perceived hand-specific disability at 3 and 6 months after surgical treatment for a hand fracture.
Secondarily, we asked if there are other patient and injury factors that are associated with these outcomes at 3 and 6 months.

\section{Patients and Methods}

In this prospective prognostic study, a total of 109 patients 18 years of age or older who had been scheduled to undergo surgery for a hand fracture between January 2013 and May 2014 were invited to participate. We defined hand fractures as including fractures of the metacarpal bone to the middle phalanx, which are associated with a higher risk of troublesome finger stiffness [1]. The patients were recruited from a tertiary care university hospital that serves as a regional emergency trauma center, and the institutional review board of the university approved this study. All patients were recruited preoperatively by a research assistant (MKC, a trained nurse) when they attended the orthopaedic outpatient clinic or when they were admitted to the ward after consenting for surgery, and all patients provided informed consent after the purpose and the procedures of this study had been explained. Surgical treatment was performed by one of two orthopaedic hand surgeons (JRB, YHR) with 16 and 9 years of orthopaedic experience, respectively, when the fracture pattern was unstable or if the fracture was intraarticular and created an unacceptable articular incongruity. Patients were excluded if they had systemic, multiorgan, or head injuries; a comorbid chronic pain condition; psychosis, bipolar disorder, or active substance dependence; or secondary gain such as from a workers' compensation dispute. Of 109 potentially eligible subjects, six subjects declined to participate in the study and five patients were excluded as a result of exclusion criteria, leaving 98 initial participants. Of those, 95 (97\%) were available at 3 months and 93 (95\%) were available at 6 months. We excluded patients lost to followup before 6-month evaluation, and 93 subjects formed the basis for all subsequent analyses (Table 1).

A power analysis indicated that a sample of 93 patients would provide $81 \%$ of the statistical power with an alpha $=0.05$ for medium effect size $\left(\mathrm{f}^{2}=0.15[6]\right)$ for linear regressions with five main predictors.

The overall severity of the injury was preoperatively evaluated with the Hand Injury Severity Scoring System (HISS) [5]. The HISS was designed to descriptively score the severity of hand injuries distal to the carpus. This score was created by assigning values for injuries to the integument (skin), skeleton (bone), motor (tendon), and nerves of each finger. The values are determined according to the area and extent of injury to each involved finger, and the sum of the values for each finger is multiplied with a constant assigned to each finger. The sum for all fingers 
Table 1. Demographic and clinical characteristics of participants

\begin{tabular}{ll}
\hline Characteristics & Number or score \\
\hline Participants & 93 \\
Mean age (years) & $45 \pm 12$ \\
$<65$ & 76 \\
$\geq 65$ & 17 \\
Male/female & $55(59 \%) / 48$ \\
& $(41 \%)$ \\
Injured side (dominant:nondominant) & $59: 34$ \\
Injury severity & \\
Minor (HISS $<20)$ & $21(23 \%)$ \\
Moderate (HISS 21-50) & $46(49 \%)$ \\
Severe and major (HISS $\geq 51)$ & $26(28 \%)$ \\
Location & \\
Metacarpal/proximal phalanx/middle phalanx & $47 / 34 / 27$ \\
Intraarticular/extraarticular & $31(33 \%) / 62(67 \%)$ \\
Open wound/closed wound & $21(23 \%) / 72(77 \%)$ \\
Treatment & \\
Open reduction and internal fixation (plating) & $24(26 \%)$ \\
Closed reduction and internal fixation & $54(58 \%)$ \\
(pinning) & \\
Closed reduction and external fixation & $15(16 \%)$ \\
Pain-coping strategies & \\
PCS & $23 \pm 8$ \\
PASS-20 & $28 \pm 14$ \\
\hline Vales & \\
\hline
\end{tabular}

Values are expressed with mean \pm SDs or number of cases (proportion [\%]); HISS = Hand Injury Severity Scoring System; PCS $=$ Pain Catastrophizing Scale; PASS = Pain Anxiety Symptoms Scale.

comprises the HISS, which ranges from 0 (no injury) to 826 (theoretically assumed maximum severity). The injuries are categorized according to severity as minor (020), moderate (21-50), severe (51-100), and major $(\geq 101)$.

The patient's pain-coping strategies were preoperatively assessed when the patients were enrolled in the study. Catastrophization was measured by using the Pain Catastrophizing Scale (PCS) questionnaire [17]. The PCS has been successfully used over a wide range of experimental and clinical settings, and it is one of the most widely used measures of this construct [10]. Sullivan et al. [22] indicated that the cutoff value for the PCS score for the "catastrophizers" versus "noncatastrophizers" is 30 points, which corresponds to the $75^{\text {th }}$ percentile (top quartile) in the data series used for validation. For the set of patients with acute pain, we determined the cutoff values for the PCS scores to be the $75^{\text {th }}$ percentile of our own data series. For our data set, the $75^{\text {th }}$ percentile cutoff value corresponded to 27 .

The pain-related anxiety was evaluated by administering the Pain Anxiety Symptoms Scale (PASS) questionnaire
[14]. A 20-item revised version of the PASS was used in the present study [13]. The PASS contains four subscales that measure the levels of various types of anxiety: $\operatorname{cog}$ nitive anxiety, fear of pain, escape and avoidance, and physiologic anxiety. The total PASS-20 score ranges from 0 to 100 , whereas the score for each five-item subscale ranges from 0 to 25 . Here, higher scores indicate greater pain-related anxiety. We determined the cutoff value for the PASS-20 scores to be the $75^{\text {th }}$ percentile in our own data series because the cutoff values for the patients with acute pain have not yet been determined or validated elsewhere. For our data set, the $75^{\text {th }}$ percentile cutoff value was 35 .

The patients returned for their functional assessment at 3 (11-14 weeks) and 6 (24-26 weeks) months after their surgical procedure. Two orthopaedic hand specialists assessed the finger motion range and grip strength, and a Quick Disability of the Arm, Shoulder, and Hand score (QuickDASH) was administered during each visit after the clinical examination. The investigators checked all returned questionnaires for completion, and the participants were assisted in completing the missing items.

The grip strength was measured by using a Jamar dynamometer (Asimow Engineering, Los Angeles, CA, USA) with the elbow flexed at $90^{\circ}$ and the forearm in a neutral rotation. The data were recorded in kilograms, and then the values were changed as a percentage of the injured side relative to the noninjured side.

The range of hand motion was evaluated by the total active ROM (TAM) of the involved rays as the sum of the arcs of active motion of the metacarpophalangeal, proximal, and distal interphalangeal joints. The relationship between a disability of the upper extremity and restricted motion has been reported to be stronger for total finger flexion than for pulp to palm measurements [11]. The range of movement for involved rays was measured with the wrist in a neutral position, and data were recorded in degrees. The values were then changed as a percentage of the injured side relative to the noninjured side.

The patient-reported outcome was evaluated with an 11item QuickDASH [2]. QuickDASH has been shown to be reliable and valid in patients with upper extremity disorders, and it demonstrated similar reliability when compared with original DASH [9].

Descriptive statistics were calculated to determine the patients' demographic and clinical characteristics. The Kolmogorov-Smirnov test was used to identify the normality of the variable distributions, and the relationships between the potential predictors (PCS, PASS, demographic variables, and injury characteristics) and the dependent variables (grip strength, TAM, and DASH score) were determined by using one-way analysis of variance or an independent $\mathrm{t}$-test for categorical predictor variables. 
Bivariate predictors were selected as candidates for the multivariate linear regression model to prevent model overfitting. The categorical variables were dummy-coded with the subgroup for the largest sample size considered as the reference group (male sex, age younger than 65 years, dominant side, minor injury severity, closed reduction and internal fixation [pinning], PCS $<27$, and PASS-20 < 35). A multiple linear regression analysis with forward stepwise variable selection was conducted with marginal significance levels for entry and removal set at 5\% and $10 \%$, respectively. This approach was selected to minimize the collinearity between the predictor variables and to determine the independent predictors of the functional recovery after hand fracture surgery. We conducted a total of six regressions with two longitudinal regressions each for grip strength, TAM, and DASH. Statistical significance was accepted at $\mathrm{p}<0.05$.

\section{Results}

Decreased grip strength was associated with catastrophic thinking (beta $=-1.29[95 \%$ confidence interval $[\mathrm{CI}],-1.67$ to -0.89$]$, partial $\mathrm{R}^{2}=11 \%, \mathrm{p}<0.001$ ) and anxiety (beta $=-0.83[-1.16$ to -0.50$]$, partial $\mathrm{R}^{2}=7 \%, \mathrm{p}=0.007$ ) at 3 months (Table 2), but by 6 months, anxiety (beta $=-0.74[-1.04$ to -0.44$]$, partial $\mathrm{R}^{2}=7 \%, \mathrm{p}=0.010$ ) but not catastrophic thinking remained an important factor for decreased grip strength. Additionally, injury severity (beta $=-0.72[-1.03$ to -0.31$]$, partial $\left.\mathrm{R}^{2}=6 \%, \mathrm{p}=0.014\right)$ and external fixation treatment (beta $=-0.64[-0.91$ to 0.37 , partial $\mathrm{R}^{2}=6 \%, \mathrm{p}=0.021$ ) were associated with decreased grip strength at 3 months but not at 6 months. Higher age, on the other hand, was an associated factor at 6 months (beta $=-0.61[-0.75$ to -0.37$]$, partial $\mathrm{R}^{2}=6 \%$, $\mathrm{p}=0.021)$. At 3 months, pain catastrophizing, anxiety, injury severity, and treatment type collectively accounted for $29 \%$ of the variance in grip strength. At 6 months, anxiety, age, and injury severity accounted for $19 \%$ of the variance.
Decreased total active ROM was associated with pain catastrophizing (beta $=-0.63[-0.90$ to -0.36$]$, partial $\mathrm{R}^{2}=6 \%, \mathrm{p}=0.024$ ) and anxiety (beta $=-0.28[-0.42$ to -0.14$]$, partial $\left.\mathrm{R}^{2}=3 \%, \mathrm{p}=0.035\right)$ at 3 months (Table 3). Also associated were injury severity (beta $=-0.81[-1.08$ to -0.54$]$, partial $\left.\mathrm{R}^{2}=10 \%, \mathrm{p}=0.001\right)$ and treatment by external fixation (beta $=-0.77[-1.11$ to $0.43]$, partial $\mathrm{R}^{2}=6 \%, \mathrm{p}=0.014$ ) or plating (beta $=-0.42$ $[-0.63$ to -0.21$]$, partial $\left.\mathrm{R}^{2}=3 \%, \mathrm{p}=0.039\right)$. However, at 6 months, only injury severity remained an associated factor (beta $=-0.88 \quad[-1.11$ to -0.65$]$, partial $\mathrm{R}^{2}=13 \%$, $\mathrm{p}<0.001)$. Similar to findings for grip strength, at 3 months, pain catastrophizing, anxiety, injury severity, and treatment type accounted for $27 \%$ of the variance in total active ROM. At 6 months, injury severity alone accounted for $13 \%$ of the variance.

Increased disability was associated with pain catastrophizing $\left(\right.$ beta $=1.09 \quad[1.39-0.79]$, partial $\mathrm{R}^{2}=12 \%$, $\mathrm{p}<0.001$ ) and anxiety (beta $=0.93$ [1.21-0.65], partial $\mathrm{R}^{2}=11 \%, \mathrm{p}=0.001$ ) at 3 months (Table 4). However, these factors failed to be associated for 6-month outcomes. Other important factors for 3-month disability were the external fixation treatment (beta $=0.79$ [1.11-0.47], partial $\mathrm{R}^{2}=7 \%, \mathrm{p}=0.014$ ) and injury severity (beta $=0.85$ [1.17-0.53], partial $\left.\mathrm{R}^{2}=8 \%, \mathrm{p}=0.011\right)$. At 6 months, injury severity (beta $=0.97[1.24-0.70]$, partial $\mathrm{R}^{2}=12 \%$, $\mathrm{p}=0.001$ ) remained a predictor of disability, and age (beta $=0.86[1.16-0.56]$, partial $\mathrm{R}^{2}=9 \%, \mathrm{p}=0.010$ ) was found to be another factor. Similar to findings for grip strength and ROM, pain catastrophizing, anxiety, injury severity, and type of treatment accounted for $38 \%$ of the variation in disability scores at 3 months. At 6 months, injury severity and age accounted for $21 \%$ of the variation.

\section{Discussion}

Although the psychological mechanisms that underlie pain have been reported to be associated with chronic pain [26],

Table 2. Independent predictor of the recovery of grip strength at each measurement time

\begin{tabular}{|c|c|c|c|c|c|}
\hline Model & Predictors & Beta $(95 \% \mathrm{CI})$ & $\mathrm{p}$ value & Partial $\mathrm{R}^{2}$ & Total $\mathrm{R}^{2}$ \\
\hline \multirow[t]{4}{*}{ Month 3} & PCS & $-1.29(-1.67$ to -0.89$)$ & $<0.001$ & $11 \%$ & \multirow[t]{4}{*}{$29 \%$} \\
\hline & PASS & $-0.83(-1.16$ to -0.50$)$ & 0.007 & $7 \%$ & \\
\hline & HISS & $-0.72(-1.03$ to -0.31$)$ & 0.014 & $6 \%$ & \\
\hline & External fixation & $-0.64(-0.91$ to -0.37$)$ & 0.021 & $6 \%$ & \\
\hline \multirow[t]{3}{*}{ Month 6} & PASS & $-0.74(-1.04$ to -0.44$)$ & 0.010 & $7 \%$ & \multirow[t]{3}{*}{$19 \%$} \\
\hline & Age & $-0.61(-0.75$ to -0.37$)$ & 0.021 & $6 \%$ & \\
\hline & HISS & $-0.62(-0.88$ to -0.36$)$ & 0.016 & $6 \%$ & \\
\hline
\end{tabular}

$\mathrm{CI}=$ confidence interval; PCS = Pain Catastrophizing Scale; PASS = Pain Anxiety Symptoms Scale; HISS = Hand Injury Severity Scoring System. 
Table 3. Independent predictor of the recovery of TAM at each measurement time

\begin{tabular}{lllrrr}
\hline Model & Predictors & Beta $(95 \% \mathrm{CI})$ & $\mathrm{p}$ value & ${\text { Partial } \mathrm{R}^{2}}^{\text {Total } \mathrm{R}^{2}}$ \\
\hline Month 3 & PCS & $-0.63(-0.90$ to -0.36$)$ & 0.024 & $6 \%$ & $27 \%$ \\
& PASS & $-0.28(-0.42$ to -0.14$)$ & 0.035 & $10 \%$ & \\
& HISS & $-0.81(-1.08$ to -0.54$)$ & $<0.001$ & $6 \%$ \\
& Treatment type & & & $3 \%$ \\
& External fixation & $-0.77(-1.11$ to -0.43$)$ & 0.039 & $13 \%$ \\
Month 6 & Plating & $-0.42(-0.63$ to -0.21$)$ & $<0.001$ & $13 \%$ \\
\hline
\end{tabular}

TAM = total active ROM; CI = confidence interval; PCS = Pain Catastrophizing Scale; PASS = Pain Anxiety Symptoms Scale; HISS = Hand Injury Severity Scoring System.

Table 4. Independent predictor of QuickDASH scores at each measurement time

\begin{tabular}{lllrrr}
\hline Model & Predictors & Beta $(95 \%$ CI $)$ & p value & Partial R $^{2}$ & Total R \\
\hline Month 3 & PCS & $1.09(1.39-0.79)$ & $<0.001$ & $12 \%$ & $11 \%$ \\
& PASS & $0.93(1.21-0.65)$ & 0.001 & $8 \%$ & $7 \%$ \\
& HISS & $0.85(1.17-0.53)$ & 0.011 & 0.014 & $12 \%$ \\
Month 6 & External fixation & $0.79(1.11-0.47)$ & 0.001 & $9 \%$ & $21 \%$ \\
& HISS & $0.97(1.24-0.70)$ & 0.010 & \\
\hline
\end{tabular}

QuickDASH = Quick Disability of the Arm, Shoulder, and Hand; CI = confidence interval; PCS = Pain Catastrophizing Scale; PASS = Pain Anxiety Symptoms Scale; HISS = Hand Injury Severity Scoring System.

the effects of these mechanisms on the outcomes of acute fractures have not yet been well investigated. Ineffective coping strategies in patients with hand fractures are likely to be associated with prolonged symptoms and disability resulting from overcautious activity restriction, resulting in posttraumatic stiffness $[16,25]$. However, the effects on functional recovery of these mechanisms after hand fractures have not been investigated in depth. The results of this study suggest that pain catastrophizing and anxiety are associated with postoperative grip strength, stiffness, and disability at 3 months after hand fracture surgery and that only anxiety is associated with decreased grip strength at 6 months.

The limitations of the study include the lack of followup data on functional outcomes beyond 6 months after surgery, although some improvements in motion and grip strength are anticipated. A healing period of 6 months after injury was chosen to not lose too many patients during followup and also because the average time until the end of healing was reported to be less than 6 months for most hand injures [23]. Second, only one type of questionnaire (QuickDASH) was used to evaluate the functional outcome of the patients. A minimal difference that is clinically important or a responsiveness of questionnaire after the hand fracture will cause differences in the functional assessments that may lead to different conclusions. Third, the patient's functional baseline state, a potential factor that influences the recovery process, could not be examined because all patients presented after sustaining a fracture. Instead, the ratios of the grip strength and the TAM between the injured and uninjured hands were obtained for each measurement. Fourth, a considerable amount of variance in the outcome measures remained unexplained. With respect to the QuickDASH scores, only $38 \%$ of the total variance was accounted for in our multivariate analysis model. Thus, other conditions may also be related to hand function and disability, including socioeconomic factors, the level of physical activity, and other aspects of psychologic morbidity that were not measured in the present study. Fifth, cutoffs for PCS or PASS used in the present study have not been validated for acute trauma. The cutoff value of the PCS score has been validated only for chronic pain [22], and continuous scores for pain anxiety and catastrophizing may limit the ability to select patients at risk within a clinical setting. Thus, we used the $75^{\text {th }}$ percentile cutoff value in our own data series. Sixth, five (5\%) patients were lost to followup before the 6-month evaluation, and there were some missing questions and questionnaires in our cohort. We used mean imputation to deal with the missing data and this method is frequently used in clinical research [8]. Finally, the patients were limited to a single ethnic population drawn from an urban area of South Korea, and therefore their characteristics and results may not generalize to other populations. 
Catastrophization and anxiety affect grip strength recovery with anxiety having persistent effects beyond 3 months. Although psychological factors are known to affect patient-reported disability more than an objective measure of function [27], considerable variances in grip strength (18\% at 3-month followup) were explained by pain-coping strategies in our multivariate analysis model. The results are consistent with the findings presented by Bot et al. [4] who noted that pain anxiety affects grip strength and disability only in patients recovering from injury, and not in healthy individuals. Grip strength appears to be a reflection of both physical impairment as well as psychological factors [27], and our results demonstrate an important role for pain coping in grip strength during early recovery from hand fractures.

ROM at 3 months is influenced by catastrophic thinking and anxiety; however, lack of coping mechanisms does not appear to have lasting effects in ROM recovery. Caution about painful movement is a normal aspect of illness behavior, but maladaptive pain perception and overcautious activity restriction negatively influence the recovery of ROM after orthopaedic injury [20]. Relationships between pain coping and ROM recovery in patients with hand fractures have not been systematically investigated, and ineffective pain self-efficacy (confidence in the ability to do things despite discomfort) was proposed as a potential predictor of finger stiffness in cases of finger ligament injuries [3]. Our results indicate that pain anxiety and catastrophic thinking in patients with hand fractures are significant predictors of hand stiffness in the early postoperative period.

Disability at 3 months, like grip strength and ROM, was influenced by catastrophic thinking and anxiety; however, lack of coping mechanisms did not affect disability at 6 months. Although there were no special intensified treatments between 3 and 6 months postoperatively, the influence of pain coping on disability was diminished at 6 months followup. The results are consistent with previous findings that psychical makeup is important in the healthy state but less important than anxiety or negative thoughts in response to pain in the context of recovery in patients with hand fractures [4]. It may be intuitive to assume that something must have gone wrong during surgery if patients do not recover well, but more severe symptoms or disability than are expected for a given physical state during early recovery may signal a patient's maladaptive nociception or ineffective coping strategy. This suggests that ineffective coping strategies could be addressed up to 3 months after sustaining a hand fracture to decrease the pain intensity and disability. In this regard, cognitive behavioral therapy, eg, exposure and acceptance therapy and relaxation training, are effective in improving coping strategies to reduce symptoms and disability for chronic musculoskeletal conditions [7]. Interestingly, age and injury severity were more important at late followup. Older patients with osteoporosis are prone to experience more severe fractures and may not be able to fully recover from their musculoskeletal injuries [18]. Conversely, only a preinjury functional state may be reflected rather than the outcome of the surgical treatment because there is no measure of the preinjury functional state. The relationship between HISS and the functional outcome after hand fracture surgery has been controversial. Some studies have reported a strong correlation between HISS and patientreported outcomes [12,19], whereas others have not shown any statistically significant correlation [15]. In the current study, HISS was associated with a decrease in QuickDASH scores up to a 6-month followup. However, statistically significant differences in outcome scores are not necessarily clinically important [21] and further analysis accounting for a minimal clinical important difference of the outcome instrument may be needed to determine whether significant associations (effects) are clinically important when making treatment decisions.

The patients' pain-coping strategies such as catastrophization and anxiety are associated with delayed recovery in patients with hand fractures, as evidenced by the scores for both the objective and subjective measures of function. Physicians and other healthcare providers may be able to improve recovery after hand fracture surgery by addressing patients' coping strategies, particularly in the first 3 months when there is the greatest correlation with objective and subjective measures of outcome. More research is needed to show whether early identification and treatment of these factors through psychological screening and cognitive-behavioral therapy will enhance the treatment outcomes in patients after acute trauma or surgical treatment.

Acknowledgments We thank M. H. Park MD, and M. K. Cha RN, for their role in data collection and J. R. Baek MD, and S. M. Rowe $\mathrm{MD}$, for advice and help in drafting the manuscript.

\section{References}

1. Barton NJ. Fractures of the hand. J Bone Joint Surg Br. 1984; 66:159-167.

2. Beaton DE, Wright JG, Katz JN, Upper Extremity Collaborative Group. Development of the QuickDASH: comparison of three item-reduction approaches. J Bone Joint Surg Am. 2005;87:10381046.

3. Bot AG, Bekkers S, Herndon JH, Mudgal CS, Jupiter JB, Ring D. Determinants of disability after proximal interphalangeal joint sprain or dislocation. Psychosomatics. 2014;55:595-601.

4. Bot AG, Mulders MA, Fostvedt S, Ring D. Determinants of grip strength in healthy subjects compared to that in patients recovering from a distal radius fracture. J Hand Surg Am. 2012;37: 1874-1880.

5. Campbell DA, Kay SP. The Hand Injury Severity Scoring system. J Hand Surg Br. 1996;21:295-298. 
6. Cohen J. Statistical Power Analysis for the Behavioral Sciences. 2nd ed. Hillsdale, NJ, USA: L. Erlbaum Associates; 1988.

7. Das De S, Vranceanu AM, Ring DC. Contribution of kinesophobia and catastrophic thinking to upper-extremity-specific disability. J Bone Joint Surg Am. 2013;95:76-81.

8. Faris PD, Ghali WA, Brant R, Norris CM, Galbraith PD, Knudtson ML; APPROACH Investigators, Alberta Provincial Program for Outcome Assessment in Coronary Heart Disease. Multiple imputation versus data enhancement for dealing with missing data in observational health care outcome analyses. J Clin Epidemiol. 2002;55:184-191.

9. Gummesson C, Ward MM, Atroshi I. The shortened Disabilities of the Arm, Shoulder and Hand Questionnaire (QuickDASH): validity and reliability based on responses within the full-length DASH. BMC Musculoskelet Disord. 2006;7:44.

10. Keogh E, Book K, Thomas J, Giddins G, Eccleston C. Predicting pain and disability in patients with hand fractures: comparing pain anxiety, anxiety sensitivity and pain catastrophizing. Eur J Pain. 2010;14:446-451.

11. Macdermid JC, Fox E, Richards RS, Roth JH. Validity of pulp-topalm distance as a measure of finger flexion. $J$ Hand Surg $B r$. 2001;26:432-435.

12. Matsuzaki $\mathrm{H}$, Narisawa $\mathrm{H}$, Miwa $\mathrm{H}$, Toishi S. Predicting functional recovery and return to work after mutilating hand injuries: usefulness of Campbell's Hand Injury Severity Score. J Hand Surg Am. 2009;34:880-885.

13. McCracken LM, Dhingra L. A short version of the Pain Anxiety Symptoms Scale (PASS-20): preliminary development and validity. Pain Res Manag. 2002;7:45-50.

14. McCracken LM, Zayfert C, Gross RT. The Pain Anxiety Symptoms Scale: development and validation of a scale to measure fear of pain. Pain. 1992;50:67-73.

15. Mink van der Molen AB, Ettema AM, Hovius SE. Outcome of hand trauma: the hand injury severity scoring system (HISS) and subsequent impairment and disability. J Hand Surg Br. 2003;28:295-299.

16. Nota SP, Bot AG, Ring D, Kloen P. Disability and depression after orthopaedic trauma. Injury. 2015;46:207-212.
17. Osman A, Barrios FX, Gutierrez PM, Kopper BA, Merrifield T, Grittmann L. The Pain Catastrophizing Scale: further psychometric evaluation with adult samples. J Behav Med. 2000;23: 351-365.

18. Roh YH, Lee BK, Noh JH, Oh JH, Gong HS, Baek GH. Factors delaying recovery after volar plate fixation of distal radius fractures. J Hand Surg Am. 2014;39:1465-1470.

19. Saxena P, Cutler L, Feldberg L. Assessment of the severity of hand injuries using "hand injury severity score", and its correlation with the functional outcome. Injury. 2004;35:511-516.

20. Schmitt MA, van Meeteren NL, de Wijer A, van Genderen FR, van der Graaf Y, Helders PJ. Patients with chronic whiplashassociated disorders: relationship between clinical and psychological factors and functional health status. Am J Phys Med Rehabil. 2009;88:231-238.

21. Sorensen AA, Howard D, Tan WH, Ketchersid J, Calfee RP. Minimal clinically important differences of 3 patient-rated outcomes instruments. J Hand Surg Am. 2013;38:641-649.

22. Sullivan MJ, Bishop SR, Pivik J. The pain catastrophizing scale: development and validation. Psychol Assess. 1995;7:524.

23. van der Molen AB, Matloub HS, Dzwierzynski W, Sanger JR. The hand injury severity scoring system and workers' compensation cases in Wisconsin, USA. J Hand Surg Br. 1999;24:184186.

24. Vranceanu AM, Bachoura A, Weening A, Vrahas M, Smith RM, Ring D. Psychological factors predict disability and pain intensity after skeletal trauma. J Bone Joint Surg Am. 2014;96:e20.

25. Vranceanu AM, Hageman M, Strooker J, Ter Meulen D, Vrahas M, Ring D. A preliminary RCT of a mind body skills based intervention addressing mood and coping strategies in patients with acute orthopaedic trauma. Injury. 2015;46:552-557.

26. Vranceanu AM, Safren S, Zhao M, Cowan J, Ring D. Disability and psychologic distress in patients with nonspecific and specific arm pain. Clin Orthop Relat Res. 2008;466:2820-2826.

27. Watson J, Ring D. Influence of psychological factors on grip strength. J Hand Surg Am. 2008;33:1791-1795. 